\title{
Evolving Four-Bars for Optimal Synthesis
}

\author{
Hans-Peter Schröcker \\ Unit Geometry and CAD \\ University Innsbruck
}

\author{
Bert Jüttler and Martin Aigner \\ Institute of Applied Geometry \\ University of Linz
}

July 6, 2010

\begin{abstract}
We present an evolution-based method for optimal mechanism synthesis. It is based on the embedding of the Euclidean motion group in the space of affine displacements upon which an object-oriented Euclidean metric is imposed. This Euclidean structure allows the use of curve and surface evolution techniques from computer aided design and image processing. We demonstrate the algorithm by synthesizing planar four-bar mechanisms and we show how to modify it so that the resulting four-bar is free of circuit defects.
\end{abstract}

Keywords: optimal mechanism synthesis, curve and surface evolution, fourbar mechanism, circuit defect.

\section{Introduction}

Optimal mechanism synthesis means finding a mechanism (type, dimension, position of base and end-effector) that approximately performs a certain task. It is a well-established field in mechanism science that evolved from the natural need for a more extensive but less accurate task description. In this article we present a novel approach to optimal motion generation.

There exists a wealth of literature on optimal mechanism synthesis and we confine ourselves to just a few references on optimal motion generation. The maybe most direct approaches use numerical techniques for solving an overconstrained system of equations [Zhang et al., 2000, Hayes et al., 2004, Smaili and Diab, 2005]. Mixed numerical and graphical methods for optimal four-bar synthesis have been presented by Yao and Angeles [2000]. They allow identification of all critical points of the underlying optimization problem. Finally, we can distinguish genetic algorithms [Cabrera et al., 2002, Kunjur and Krishnamurty, 1995, Zhou and Cheung, 2001] and methods based on evolution techniques, for example Shiakolas et al. [2002], 2005.

Our approach fits into the last category. Its main distinction from other optimal synthesis schemes is the use of an object-oriented metric that makes the design space Euclidean. This allows the advantageous use of geometric techniques 
from computer aided design and image processing. In particular, we perform closest point computation and curve/surface evolution in this space. Evolution techniques permit great flexibility and control over the mechanism shape during the evolution and can be used to avoid mechanism defects. Moreover, the formulation of the synthesis problem within this framework is independent of the fixed coordinate frame. While our concept is general and can, at least in principle, be used for synthesizing many mechanisms of diverse type and shape, we confine ourselves to the synthesis of planar four-bars. Extensions to further mechanism types will be discussed in a subsequent publication.

In Section 2 we introduce the object-oriented metric used in our approach. It is followed by an outline of a general framework for curve and surface fitting via evolution in Section 3. In Section 4 we explain how to formulate a fourbar synthesis problem within this framework and present a first example. In Section 5 we discuss how to incorporate additional constraints in order to avoid unwanted defects of the synthesized four-bars and in Section 6 we conclude the paper with directions for future research.

\section{A metric for affine displacements}

Measuring the "distance" between two displacements is a fundamental problem of mechanism science with many important applications, in particular in mechanism synthesis. Among the various available concepts (see for example Larochelle et al. [2004], Angeles [2006]) we choose an approach which leads to an embedding of the Euclidean motion group into the space of affine displacements that is equipped with an object-oriented Euclidean metric [Belta and Kumar, 2002, Chirikjian and Zhou, 1998]. This metric has already been used for motion design [Hofer et al., 2004, Hofer and Pottmann, 2004], for computing generalized penetration depths [Nawratil et al., 2007], and for defining performance indices of robots [Nawratil, 2007], but apparently not in mechanism design.

The distance concept is based on the choice of a number of feature points $m_{i} \in \mathbb{R}^{2}$. These feature points define a positive definite scalar product $\langle\alpha, \beta\rangle=$ $\sum\left\langle\alpha\left(m_{i}\right), \beta\left(m_{i}\right\rangle\right.$ and an associated squared distance $\operatorname{dist}^{2}(\alpha, \beta)=\sum \| \alpha\left(m_{i}\right)-$ $\beta\left(m_{i}\right) \|^{2}$ between $\alpha$ and $\beta$ that depends only on the barycenter of the feature points and on their inertia matrix. It endows the space $\mathrm{GA}^{+}(2)$ of affine displacements with a Euclidean metric. The space $\mathrm{GA}^{+}(2)$ contains the Euclidean motion group $\mathrm{SE}(2)$ as a sub-manifold of dimension three. The feature points $m_{i}$ can be replaced by an arbitrary mass distribution $\mu$ on $\mathbb{R}^{2}$. In this case the formulas for inner product and distance are expressed in terms of integrals [Chirikjian and Zhou, 1998]. The inner product defined by the feature point $m_{i}$ (or by the mass distribution $\mu$ ) is also generated by four suitable chosen points of equal mass. Hence, the computation of inner product and distance is very efficient. Extensions of this distance concept to higher dimensions are straightforward. 


\section{Evolution-based fitting of algebraic varieties}

Now we describe a general concept for fitting implicitly defined varieties to a number of prescribed feature points (see also Aigner and Jüttler [2007] and Aigner et al. [2007]). Its relation to optimal synthesis of four-bars will be explained in the next section.

Consider a system $F_{1}(x), \ldots, F_{m}(x)$ of polynomials and its zero set $C \subset \mathbb{R}^{d}$. We assume that the coefficients of $F_{i}$ depend on a vector $s=\left(s_{1}, \ldots, s_{p}\right)$ of shape parameters. The vector $s$ of shape parameters is to be determined such that the zero set $C$ of the polynomials $F_{i}$ approximates a set of target points $q_{0}, \ldots, q_{n}$. By $p_{j}$ we denote the point on $C$ that minimizes the distance to $q_{j}$ and by $\nabla F_{i}$ we denote the gradient of $F_{i}$ with respect to $x$.

Now we view $s$ as depending on a time-variable $t$, that is $s=s(t)$. As $s(t)$ varies in time, the points $p_{j}=p_{j}(t)$ move along certain trajectories. Because of $F_{i}\left(p_{j}(t), s(t)\right) \equiv 0$ we also have

$$
\frac{d}{d t} F_{i}\left(p_{j}(t), s(t)\right)=\left\langle\nabla F_{i}, \dot{p}_{j}\right\rangle+\sum_{l=1}^{p} \frac{\partial F_{i}}{\partial s_{l}} \dot{s}_{l} \equiv 0
$$

where the derivatives of $F_{i}$ are evaluated at $\left(p_{j}(t), s_{j}(t)\right)$ and the dot denotes derivatives with respect to $t$. Hence, $\dot{p}_{j}$ is the velocity of the closest point $p_{j}$. By $\left\{b_{j 1}, \ldots, b_{j m}\right\}$ we denote an orthonormal basis of the normal space of $C$ in $p_{j}$. Ideally, the change of $s(t)$ should be such that $q_{j}-p_{j}=\dot{p}_{j}$, that is,

$$
\left\langle q_{j}-p_{j}, b_{j k}\right\rangle=\left\langle\dot{p}_{j}, b_{j k}\right\rangle \text { for } j=0, \ldots, n \text { and } k=1, \ldots, m \text {. }
$$

There exist coefficients $\beta_{j k i}$ such that $b_{j k}=\sum_{i=1}^{m} \beta_{j k i} \nabla F_{i}$. In view of $(1)$, this yields

$$
\left\langle\dot{p}_{j}, b_{j k}\right\rangle=\sum_{i=1}^{m} \beta_{j k i}\left\langle\dot{p}_{j}, \nabla F_{i}\right\rangle=-\sum_{i=1}^{m} \beta_{j k i} \sum_{l=1}^{p} \frac{\partial F_{i}}{\partial s_{l}} \dot{s}_{l}
$$

Hence, (2) leads to an over-constrained system of linear equations in the unknowns $\dot{s}_{j}$ that can be solved in least-squares sense. The result is independent of the choice of the basis vectors $b_{j k}$. Following the thus defined flow in the manifold of shape parameters leads to a locally optimal solution. It can be shown that the numerical integration of the trajectories via explicit Euler steps is equivalent to a Gauss-Newton method for non-linear least-squares optimization.

\section{A concept for optimal four-bar synthesis}

The evolution technique for fitting algebraic varieties to points as described in Section 3 can be used for optimal mechanism synthesis. In principle it is applicable for arbitrary mechanisms (planar, spherical, spatial, serial, or parallel). We are going to describe the concept for the case of planar four-bar synthesis. Extensions to other classes of mechanisms are postponed to a further publication. 

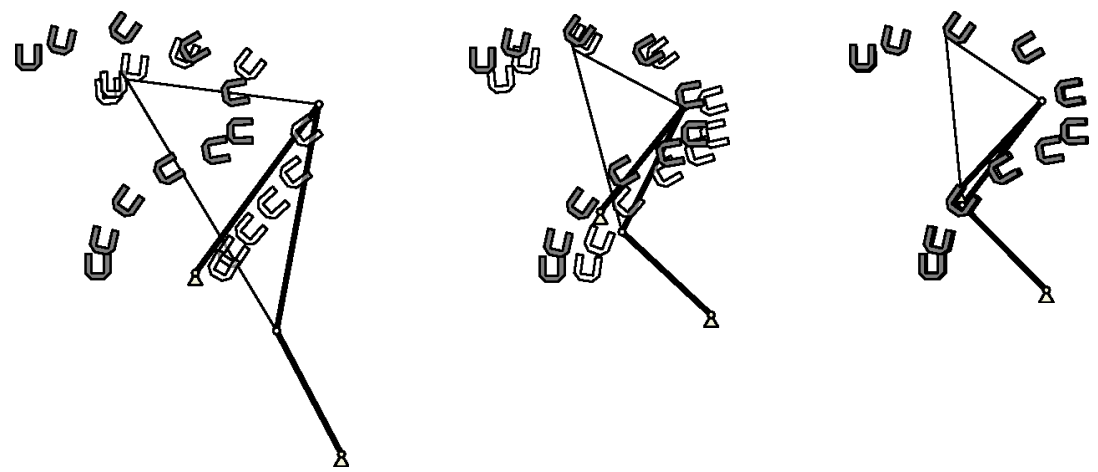

Figure 1: Solution to McCarthy's eleven poses (from left to right: initial fourbar, after 15 iterations, after 30 iterations)

We model a four-bar motion via two circle constraints. For $i=1,2$ the point with coordinates $\left(a_{i}, b_{i}\right)$ in the moving system is constraint to a circle with center $\left(\xi_{i}, \eta_{i}\right)$ and radius $\varrho_{i}$. Then the synthesis problems relates to the concept of Section 3 as follows:

- The target points $q_{0}, \ldots, q_{n}$ are $n+1$ prescribed poses, viewed as points on the Euclidean motion group $\mathrm{SE}(2) \subset \mathrm{GA}^{+}(2) \subset \mathbb{R}^{6}$.

- The vector $s$ of shape parameters has the ten entries $a_{1}, b_{1}, \xi_{1}, \eta_{1}, \varrho_{1}, a_{2}$, $b_{2}, \xi_{2}, \eta_{2}$, and $\varrho_{i}$.

- The four-bar motion is defined by five algebraic equations $F_{1}, \ldots, F_{5}$. Three of them (the orthogonality conditions on the transformation matrix) define $\mathrm{SE}(2)$ as sub-manifold of $\mathrm{GA}^{+}(2)$ and remain constant over time. The two remaining equations are the circle constraints $\| \alpha\left(a_{i}, b_{i}\right)-$ $\left(\xi_{i}, \eta_{i}\right) \|^{2}=\varrho_{i}^{2}$.

- All distances and angles in $\mathbb{R}^{6}$ are measured using the inner product $\langle.,$.$\rangle ,$ which corresponds to the feature-point dependent metric, as described in Section 2. Also, the gradient vectors are computed with respect to that metric.

As a first example we fit a four-bar to eleven prescribed poses, proposed by J. Michael McCarthy on the occasion of the ASME Design Engineering Technical Conference in Montreal in 2002 [see also Chen and Angeles, 2007]. Figure 1 shows these poses, the initial configuration, and the results after 15 and 30 iterations. In each picture we display the target poses (dark), the closest poses of the current four-bar motion (white), and the four-bar mechanism in a configuration to reach the third of the prescribed poses. As can be seen, the prescribed poses are particularly amenable to a four-bar approximation.

In all examples of this article we choose the mass distribution by defining a set $M$ of feature points that roughly represents the shape of the end-effector as 
depicted in Figure 1. The total mass distribution is the union of all images of $M$ under the prescribed poses.

In order to control the step-size in the evolution sequence, we specify a certain maximum allowed displacement $H$ for the closest points $p_{j}$. The squared length of the displacement vector of $p_{j}$ approximately equals $\delta_{j}^{2}=\sum_{k=1}^{m}\left\langle\dot{p}_{j}, b_{j k}\right\rangle^{2}$ and can be computed via (3). The allowed step size $h$ for the current step is obtained as $h=H \min \left\{\delta_{j}^{-1}\right\}$. Furthermore, we decrease the value of $H$ once the maximal expected change of the closest points is smaller than $H$ in order to avoid forcing a large step away from an already good configuration.

Currently, the most time-consuming part of the evolution procedure consists in the computation of the closest points on the constraint variety in $\mathrm{GA}^{+}(2)$. The remaining calculations (evaluations of elementary functions and solving a small linear system in the least-squares sense) are relatively cheap. The closest point computation is equivalent to computing the closest pose on a four-bar motion to a given pose. In our implementation we compute these points via standard constrained optimization routines. This is not completely reliable and we have no guarantee that we really end up in the global minimum. We found, however, that using the closest points from the preceding iteration step as starting values for the new closest points yields a reasonably good performance. A single closest point computations takes about 1-2 ms.

\section{$5 \quad$ Penalty functions}

In this section we show how to integrate penalty functions into our evolution framework. We discuss how to avoid singular four-bars and use this technique to produce optimal solutions that are free of circuit defects. Denote the base length of the four-bar by $g$ and the coupler length by $h$. The lengths of driver and follower are the shape parameters $\varrho_{1}$ and $\varrho_{2}$, respectively, while $g$ and $h$ are simple functions of the remaining shape parameters. Then a four-bar is singular (folding) if and only if one of the functions

$$
C_{1}=\frac{\left(\varrho_{1}+\varrho_{2}-g-h\right)^{2}}{\left(\varrho_{1}+\varrho_{2}+g+h\right)^{2}}, \quad C_{2}=\frac{\left(\varrho_{1}-\varrho_{2}+g-h\right)^{2}}{\left(\varrho_{1}+\varrho_{2}+g+h\right)^{2}}, \quad C_{3}=\frac{\left(\varrho_{1}-\varrho_{2}-g+h\right)^{2}}{\left(\varrho_{1}+\varrho_{2}+g+h\right)^{2}}
$$

vanishes. For each of the functions $C_{i}$ we choose a minimal permissible value $\delta_{i}$, and an offset $\sigma_{i}$. Using these values, we construct a smooth penalty function $w_{i}$ and an activator function $\alpha_{i}$ as depicted in Figure 2. If $C_{i}$ falls within $\left[\delta_{i}, \delta_{i}+\sigma_{i}\right]$, then the evolution pushes the shape parameters away from the zero set of $C_{i}$. This is achieved by adding the equation

$$
\alpha\left(C_{i}\right) \cdot\left(\sum_{l=1}^{10} \frac{\partial C_{i}}{\partial s_{l}} \dot{s}_{l}-w_{i}\left(C_{i}\right)\right)=0,
$$

which is linear in $\dot{s}$, to the over-constrained system obtained from (3).

A four-bar mechanism is called circuit defective with respect to a set of poses, if it cannot attain the poses within the same continuous range of motion Chase and Mirth [1993]. It needs to be disassembled and re-configured in a 

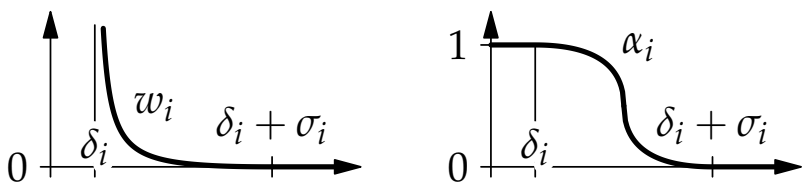

Figure 2: Penalty function $w_{i}$ and activator function $\alpha_{i}$

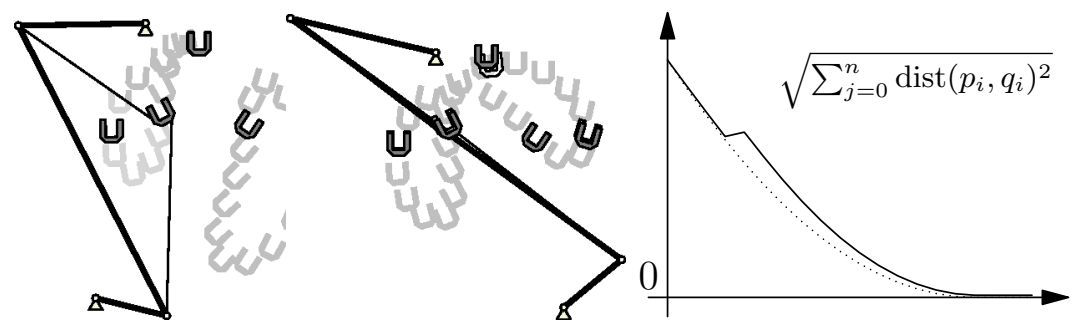

Figure 3: Avoiding solutions with circuit defect.

different way in order to reach all poses. In our case, the poses are only attained approximately. Therefore, we say that a four-bar exhibits a circuit defect, if it is circuit defective with respect to the closest poses.

Assume now that the optimal four-bar obtained via evolution is circuit defective but the initial curve that evolves towards the target was not. Since the closest points $p_{i}(t)$ move smoothly during the evolution process, this can only happen if the topology of the four-bar motion - which is seen as a curve on $\mathrm{SE}(2) \subset \mathrm{GA}^{+}(2)$ - changes to two disconnected components (either from one or two components, or from two components via one component back to two components). At the transition between any two stages is a folding four-bar. Therefore, four-bars with circuit defect can be prevented by avoiding folding four-bars. A similar technique can also avoid Grashof defects and mechanism with a bad transmission ratio.

An example avoiding a circuit defect is shown in Figure 3. The five prescribed poses permit precisely one exact solution that suffers from a circuit defect. The direct application of our algorithm (left-hand side) produces this defective mechanism. The picture in the middle shows an optimal solution, generated from the same initial values, but avoiding the assembly mode defect. The graph on the right-hand side displays the evolution of the square root of the sum of squared distances between target points and closest points over time - dotted for the first (assembly mode defective) solution, and in continuous line-style for the second solution. As opposed to the first solution's graph, the graph of the second solution comes only close to zero. The kink occurs when the four-bar is pushed away from a singular configuration. 


\section{Conclusion}

We presented an algorithm for optimal mechanism synthesis (motion generation). In this paper we discussed the synthesis of a planar four-bars. The extension to other classes of mechanisms and incorporation of further defect-avoiding constraints is subject of ongoing research.

The evolution viewpoint to numerical optimization - as described in this paper - provides several advantages, including the connection to well-established techniques from image processing, the availability of a geometrically motivated step-size control, the possibility to add constraints via penalty functions, and the invariance with respect to the specific choice of the shape parameters [see Aigner and Jüttler, 2008]. In addition our method provides a geometrically significant solution, due to left-invariance of the used metric and the repeated closest point computation.

\section{References}

Martin Aigner and Bert Jüttler. Approximation flows in shape manifolds. In P. Chenin, T. Lyche, and L. L. Schumaker, editors, Curve and Surface Design, Avignon 2006, pages 1-10. Nashboro Press, 2007.

Martin Aigner and Bert Jüttler. Gauss-newton-type techniques for robustly fitting implicitly defined curves and surfaces to unorganized data points. FSP Report Industrial Geometry 65, University Linz, 2008. To appear in Proc. Shape Modelling International 2008, IEEE; available at http://www.ig.jku.at.

Martin Aigner, Zbyněk Šír, and Bert Jüttler. Evolution-based least-squares fitting using pythagorean hodograph spline curves. Comput. Aided Geom. Design, 24(6):310-322, 2007.

Jorge Angeles. Is there a characteristic length of rigid-body displacements? Mech. Machine Theory, 41(8):884-896, 2006.

Calin Belta and Vijay Kumar. An SVD-based projection method for interpolation on se(3). IEEE Trans. Robotics Automation, 18(3):334-345, 2002.

J. A. Cabrera, A. Simon, and M. Prado. Optimal synthesis of mechanisms with genetic algorithms. Mech. Machine Theory, 37(10):1165-1177, 2002.

T. R. Chase and J. A. Mirth. Circuits and branches of single-degree-of-freedom planar linkages. ASME J. Mechanical Design, 115(2):223-230, 1993.

C. Chen and J. Angeles. Kinematic synthesis of an eight-bar linkage to visit eleven poses exactly. In Proc. CDEN/C2E2 2007 Conference, Winnipeg, Alberta, 62007.

G. S. Chirikjian and Shiyu Zhou. Metrics on motion and deformation of solid models. ASME J. Mechanical Design, 120(2):252-261, 1998. 
M. John D. Hayes, Tim Luu, and Xiao-Wen Chang. Kinematic mapping application to approximate type and dimension synthesis of planar mechanisms. In J. Lenari and C. Galletti, editors, Advances in Robot Kinematics. Kluwer Academic Publishers, 2004.

Michael Hofer and Helmut Pottmann. Energy-minimizing splines in manifolds. Transactions on Graphics, 23(3):284-293, 2004. Proc. of ACM SIGGRAPH 2004.

Michael Hofer, Helmut Pottmann, and Bahram Ravani. From curve design algorithms to the design of rigid body motions. The Visual Computer, 20(5): 279-297, 2004.

A. Kunjur and S. Krishnamurty. Genetic algorithms in mechanism synthesis. In Fourth Applied Mechanisms and Robotics Conference AMR 95-068, pages 1-7, Cincinnati, Ohio, 1995.

Pierre M. Larochelle, Andrew P. Murray, and Jorge Angeles. SVD and PD based projection metrics on $\mathrm{SE}(\mathrm{n})$. In J. Lenari and C. Galetti, editors, On Advances in Robot Kinematics, pages 13-22. Kluwer Academic Publishers, Dordrecht and Boston and London, 2004.

Georg Nawratil. New performance indices for $6 \mathrm{R}$ robots. Mech. Machine Theory, 42(11):1499-1511, 2007.

Georg Nawratil, Helmut Pottmann, and Bahram Ravani. Generalized penetration depth computation based on kinematical geometry. Geometry Preprint Series 172, Vienna University of Technology, 2007.

P. S. Shiakolas, D. Koladiya, and J. Kebrle. On the optimum synthesis of four-bar linkages using differential evolution and the geometric centroid of precision positions. J. Inversive Problems Engineering, 10(6):485-502, 2002.

P. S. Shiakolas, D. Koladiya, and J. Kebrle. On the optimum synthesis of six-bar linkages using differential evolution and the geometric centroid of precision positions technique. Mech. Machine Theory, 4(3):319-335, 2005.

Ahmad A. Smaili and Nadim A. Diab. Optimum synthesis of mechanisms using tabu-gradient search algorithm. ASME J. Mechanical Design, 127(5):917-923, 2005 .

Jin Yao and Jorge Angeles. Computation of all optimum dyads in the approximate synthesis of planar linkages for rigid-body guidance. Mech. Machine Theory, 35(8):1065-1078, 2000.

Xiong Zhang, Ji Zhou, and Yingyu Ye. Optimal mechanism design using interior-point methods. Mech. Machine Theory, 35(1):83-98, 2000.

H. Zhou and H. M. Cheung, Edmund. Optimal synthesis of crank-rocker linkages for path generation using the orientation structural error of the fixed link. Mech. Machine Theory, 36(8):973-982, 2001. 